\title{
Eysenck's Personality Traits (EPQ-R) Score Differences Based on Gender and Role as Victim or Perpetrator of Domestic Violence in Uganda
}

\author{
Wilber Karugahe \\ Department of Mental Health \& Community Psychology, Makerere University, Kampala, Uganda
}

Email address:

wkarugahe@hotmail.com

\section{To cite this article:}

Wilber Karugahe. Eysenck's Personality Traits (EPQ-R) Score Differences Based on Gender and Role as Victim or Perpetrator of Domestic Violence in Uganda. Psychology and Behavioral Sciences. Vol. 8, No. 5, 2019, pp. 119-127. doi: 10.11648/j.pbs.20190805.13

Received: September 5, 2019; Accepted: October 5, 2019; Published: October 17, 2019

\begin{abstract}
The present study aims to study Eysenck's personality traits subscales (Psychoticism, Extroversion and Neuroticism) and how these traits differ among victims and perpetrators of domestic violence of both gender ( $\mathrm{N}=120)$. A two by two MANCOVA was conducted and the results show no statistical significant differences, no interaction between males and females, victims and perpetrators on all Psychoticism, Extroversion and Neuroticism while controlling lie as covariate. However through inspecting mean scores, perpetrators more than victims reported slightly higher scores on Neuroticism (implying that they are anxious, depressed and react slowly to aversive stimuli but also have high levels of stability); and scored high on psychoticism (implying that higher scorers are considered aggressive, antisocial, cold and egocentric). In contrast, victim's results revealed that irrespective of gender, victims more than perpetrators had high scores on extraversion. Higher scores on extroversion implies that females are more social, lively and sensation seeking. Indeed, majority of the victims were females and it's not surprising that African women despite her being victims, could still afford to smile (scored high on extroversion). Thus, this study informs counsellors, social workers, psychologists and other helping professionals the need for gender inclusivity in handling cases of domestic violence but also being aware of victims/perpetrators individual's personality differences. In addition, being aware of victims and perpetrators personality traits scores differences is informative and helps professionals to predict that person's likely future behavior and how to respond.
\end{abstract}

Keywords: Gender, Role, Personality Trait, Victim, Perpetrator

\section{Introduction}

To the lay person, personality is judged in a social context, such as how well people get on with others and their style of interacting as well as their appearance [1]. However, the purpose of studying personality using Eysenck's Personality Traits (EPQ-R) among victims and perpetrators of both gender is to explore the possibility of conditioning individuals associate antisocial behaviors with punishment which produces anxiety to repeat or re-engage in antisocial behaviours. Where this is successful even thinking of antisocial behaviours produces anxiety among individuals with particular traits and therefore the person avoids being antisocial and/or perpetrating violence. Indeed, this notion of trait predicting behaviour continues to influence and be the view of prominent trait theorists such as [2], who stated that:
"Traits cannot be directly observed but rather must be inferred from patterns of behaviour and experiences that are known to be valid trait indicators".

However, human beings are all different with unique personality characteristics so that not even identical twins will have exactly same personal traits [3]. Some of these personality traits may be observable or unobservable and conscious or unconscious (e.g., unobservable aspects are thoughts, memories and dreams, while behaviours are the individual's physical, social, mental, emotional actions and many more behaviours are observable through overt actions [4]. Indeed, [5] have argued that the term trait in personality refers to broad traits or dimensions found in the big five factor model: neuroticism, extraversion, openness, agreeableness, consciousness [6-7] and Eysenck's personality theory (gigantic three: neuroticism, extroversion and 
psychoticism [5] but each broad trait comprises narrow traits which are correlated e.g. people with a tendency towards one of them have a tendency towards others. Next, big five factor and gigantic three are discussed accordingly.

Firstly, trait and factor theories-big five factor model measured by NEOPI-R, which is traced from Allport, (1935) list of English language trait names, led to a sixteen personality factor questionnaire scale (16 PF with three domains NEO), which was later developed to NEOPIR and is still being widely used [5]. In this model, previous research has clustered personality traits into five basic traits (Big 5): Neuroticism (N) - which is the opposite of emotional stability and people with high scores on $\mathrm{N}$ often experience guilt and low self-esteem; extroversion (E) - people with high scores on $\mathrm{E}$ are viewed as being social and assertive in life; openness to experience $(\mathrm{O})$ - people scoring high on $\mathrm{O}$ are open minded, high on imagination and they also have independent judgement; agreeableness (A) - high scores tend to be tolerant, trusting and they value other people's beliefs; and finally conscientiousness (C) scale - high scorers tend to be achievement oriented, they distinguish themselves for trustworthiness and are responsible [8].

For one to understand how the personality traits link to human criminal actions or violent behaviours, [9] recommends that one must gain theoretical insight. The trait and factor theorists point out that human beings are different from other animals because of their ability to report data about themselves [6]. It is further argued that people are able to evaluate themselves and render reasonably reliable reports concerning their attitudes, temperament, needs, interests and behaviours. More importantly, psychologists behind trait and factor theory emphasize that genetic factors of personality are inherited and of biological components and influence human behaviours but social factors do not [6]. Critics of this theory such as [10], indicate that its failure to recognize the role of social factors in influencing human behaviour in preference to biological factors is a weakness. It is argued further by [10] who questioned whether persons possessed stable personality traits definite that produce same behaviour all the time. It is important to note, however, [10] did not say people are completely inconsistent. There are cases such as aggression and violence in which people who are prone to violence attack others only when the given situation has a certain meaning for them, such as when they regard themselves as being threatened or criticized [10]. Indeed, this article perceives personality traits in relation to domestic violence role of being victim or perpetrator which might occur when men's position, power and control are threatened or questioned. Also, female violence to men (victims) has been found to be a result of perceived threat to their life and opting to fight back/ retaliation [10-11]. Hence, [10] concludes that essentially some people indeed opt to act the same way whenever an aggressive/violent opportunity arises and if these individuals are free to do what they want in a given situation, there is good chance that the individual will behave in the same manner on many occasions.

Overall, the big five are marred in controversy. For example, scholars have argued that openness is not viewed to be different from intellect and others have questioned whether openness should be ranked as a factor in the big five [5]. Although the big five are criticized as containing too many traits to describe personality and being an unstable measure across different cultures, the NEO-PI is still being used. This is partly because it is related to Eysenck's Personality Inventory [13] which is one of the most used psychometric tools in research on personality [8]. Hence, Eysenck's Personality Questionnaire (EPQ shorter version by [14], is used and it is critically evaluated next to rationalize and situate this study.

Secondly, the personality theory of Eysenck, states there are three broad personality factors (gigantic three) i.e. extroversion, neuroticism and psychoticism. These traits are assessed in a self-report questionnaire (Eysenck Personality Questionnaire), which includes yes/no answers a lie scale intended to measure the subject's tendencies to lie when answering questions [5]. Throughout the years this questionnaire has been revised, resulting in several different versions, [13]. Although Eysenck's higher order dimensions are intended not to be correlated, there are slightly positive correlations between male subjects and the other two scales [5]. These gigantic three traits according to Eysenck [5] are: Extroversion personality trait: high scorers are considered sociable, lively and sensation seeking and low scorers are regarded to be low in arousal and in need of environmental stimulation; Neuroticism personality trait: people who score high on neuroticism are anxious, depressed and react strongly to aversive stimuli- high scorers are also regarded as having high levels of instability whereas low neuroticism scorers are considered stable and relatively unreactive; Psychoticism personality trait: people who score high on psychoticism are considered aggressive, antisocial, cold and egocentric and low scorers are social and warm. Eysenck concluded that more people have moderate extroversion, neuroticism and psychoticism and extreme scorers are rare and hard to find because most people often score moderately on personality. Furthermore, Eysenck emphasised that both neurotic and psychotic traits are normal personality traits even though they might predispose a person to neurotic and psychotic disorders (in a very few individuals). Finally, Eysenck emphasised that it is the normal network in which a dimension is embedded that provides its validity (e.g., the network must specify the psychometric properties of a dimension but also its cultural variance [5].

Thirdly, irrespective of wide research on personality, questions remain; For example, is the 'five trait factor' or Eysenck's 'gigantic three structures' universally applicable to both men and women, victims and perpetrators, and in all cultural settings or, alternatively, do these traits reflect ideas about personhood that are limited to the west, where the studies from which these approaches have been derived have been conducted? To answer these questions previous research is reviewed through the lens of trait and the big five factor model and Eysenck's gigantic three traits. For instance, [14] large study among 50 different cultures from all 
continents except Antarctica used the big five factor model to assess trait adjectives. Although in many of the locations studied, the factor structure of the big five was replicated, in most developing countries (e.g., Botswana, Ethiopia and Uganda amongst others) the factor structure was not so evident. Furthermore, the quality of the data collected was poor, suggesting that people did not understand the questions or were unfamiliar with answering questions in that format. Hence, this thesis adopts Eysenck's personality questionnaire, a much shorter version but also widely and previously used in Uganda to study gender [15]. From personality theory, Eysenck developed his personality theory of offending, which emphasises socialisation by viewing criminogenic behaviour as developmentally immature, selfish and seeking to achieve immediate gratification [16]. Eysenck argued that the process of socialisation is where the individual is taught the ability to delay gratification and be socially oriented through conditioning and immaturity. Going against the norm is accompanied with a severe punishment that makes individuals associate anxiety with antisocial behaviours. Where this is successful, even thinking of antisocial behaviour produces anxiety and therefore the person avoids being antisocial or committing crime. Eysenck concluded that individuals who scored high on neuroticism and psychoticism had nervous systems making it hard to condition them and as a result would not learn easily to respond to anxiety; hence they would be more likely to act antisocially [16]. This theory has been criticized for its failure to address the concerns of forensic psychologists about why perpetrators do what they do [9]. Despite this criticism, [9] argues that this theory can tell us whether the perpetrator is extrovert, neurotic or psychotic; which then informs the professional practice regarding conditioning.

Indeed, there are few studies globally and none in Uganda that have studied personality traits through the lens of victims and perpetrators for both genders, which this study aims to achieve. This study aims to answer the following research question and hypothesis.

\subsection{Objectives of the Study}

To establish if there are gender (male or female) and role (victim or perpetrator) differences and interactions in relation to Eysenck's personality traits.

\subsection{Hypothesis of the Study}

There are no significant gender and role differences-effect in sub-scales of Eysenck's personality traits in a Ugandan sample.

\section{Research Methods}

\subsection{Population of Interest and Sample Size}

All victims and perpetrators irrespective of their gender from organizations-institutions studied were of interest to this study. This study recruited 120 Ugandans, male and female not partnered who had experience in coercive behaviours including domestic violence. Eligible participants were accessing conflict resolution and counselling services at Police Unit (CFPU) within Kampala Capital City. The study also recruited victims who sought care at a specialized service Non-Government Organization in Kampala Capital City.

\subsection{Sampling}

Purposive and convenient voluntary sampling strategies were used because of specific required participant characteristics [17]. Voluntary sampling was further used due to the sensitivity of the topic and to ensure that individuals were not coerced to participate but rather participated voluntarily (see ethical considerations in 2.5).

\subsection{Procedures of Recruitment}

Male and Females not partnered who had experience in coercive behaviours including domestic violence were recruited from people accessing conflict resolution and counselling services at Police Unit (CFPU) and victims who sought care at a specialized service Non-Government Organization in Kampala Capital City. Self-identification was used to identify victims and perpetrators. With help of police administration and manager, researcher was responsible for data collection. The questionnaires were answered by participants and returned immediately. Data was locked in safe lockers for confidentiality before embarking on data entry and analysis.

\subsection{Research Tools}

1) Role in violence and Gender of participants were answered along other personal data informationcharacteristics of participants: These include: gender (male or female), role (victim or perpetrator) and employment status.

2) Eysenck Personality Traits (EPQ-Short German Version) was used to measure personality traits [13]. The questionnaire consists of three measures conforming to three personality traits (Psychoticism, Extroversion and Neuroticism) in Eysenck's 1990 theory, plus a lie scale. There are 12 items in each of the four scales with dichotomous binary responses of yes and no scored as 1 and 0 respectively. The maximum possible score on each original sub-scale is 12 or 0 with 2 items reverse coded in extroversion, 7 in psychoticism and 9 in lie; the neuroticism scale had no reversed items. A pilot test was done with 20 participants and Cronbach's alpha scores for each sub-scale were: .72 for Extroversion, .75 for Neuroticism, .71 for Psychoticism, .70 for Lie. The items that were not significant were deleted (items 31, 28 and 26 from psychoticism; items 25 and 21 from Neuroticism; items 19 and 23 from Extroversion; items 37 and 24 from the lie scale). The lie scale lists behaviours that are socially desirable but infrequently practiced or frequently practiced but socially undesirable [18]. Indeed, the lie 
scale was originally introduced to detect those who fake being good [19] by diagnosing a set of rare acts being endorsed by the respondent while frequently performed non-desirable acts are being denied. There is a large body of research evidence that individuals with high motivation to fake being good on lie scale scores suppress their own neurotic scores, which leads to a negative correlation between lie and neuroticism scales [19]. Although the lie scale is open to multiple interpretations, some researcher's urge, with evidence, that it should interpreted as measuring personality dimensions in their own right [19]. Although the lie scale is open to multiple interpretations [19], this current study has interpreted and treated lie scale as a covariate variable to detect whether or not elevated high lie scores indicate one faking to be good or not.

\subsection{Ethical Considerations}

Firstly, Ethical approval was sought from the University of Huddersfield-UK and consequently approval and clearance was gained from the School Research and Ethics Panel (SREP).

Secondly, at the national level within Uganda where data was collected, the study sought and gained approval and clearance from the Institution Review Committee Board Clearance (IRCB) and the Uganda National Council of Science and Technology (UNCST). This approval/clearance was done in accordance with a Ugandan government regulation established in 1990 that requires any research to have research clearance and permission before any data is collected from human beings [20]. This is to ensure i) the safeguarding of people against potentially harmful scientific activities; ii) the assessment of the credibility and skills of the researcher, to ascertain their ability to collect data without causing harm to participants; iii) the protection of the welfare of human research objects through science and technology regulations, guidelines and monitoring compliance [20]. Thirdly, the study gained permission from the organisations/institutions where data was collected. This involved seeking the manager's permission from NonGovernment Organisations (NGOs) and the Office of
Commissioner Child and Protection Unit (UP-CFPU). Following successful ethical approvals, pilot test was done on instruments for validation purposes using 20 participants. In addition, participants were assured confidentiality, participant's rights to withdrawal without any conditions and were not required to write their names for confidentiality purposes during data collection process.

\subsection{Statistical Analysis}

Data was sorted, coded and entered into Statistical Package for Social Scientists (SPSS/PASW version 20). The data was screened for normality using Kolmogorov-Smirnov not Shapiro-Wilk because the number of participants was over 50. The results were normally distributed within the sample from which it was collected, hence used parametric tests rather than non-parametric which are less powerful [2122]. In addition, statistical power refers to the ability of a test to find an effect that genuinely exists; thus, since nonparametric tests are less powerful, this implies that if there is a genuine effect in the data, then a parametric test is more likely to detect it than a non-parametric one [21]. Thus, the use of a parametric test avoids false negative (type 11 error) of accepting that there is no difference between groups when, in reality, a difference exists [21]. The independent sample ttest was used to analyse the data. Consequently, descriptive statistics were used to analyse demographic characteristics (frequencies and percentages) and MANCOVA was used to test the $2 \times 2$ relationships, differences and interactions or effect.

\section{Results}

\subsection{Demographic Characteristics}

As in most academic studies, participants' demographic characteristics are very important and give context to the data. The description of demographic characteristics further gives the study a sense of authority; confidence and credibility, as the source of the data and the results are evident. For this study the demographic characteristics of participants are as follows.

Table 1. Demographic Characteristics $(N=120)$.

\begin{tabular}{llll}
\hline Personal Bio data & Response & Frequency & Percent \\
\hline \multirow{2}{*}{ Gender } & Male & 41 & 34.2 \\
& Female & 79 & 65.8 \\
Role & Victim & 60 & 50.0 \\
& Perpetrator & 60 & 50.0 \\
\multirow{2}{*}{ Employment status } & Unemployed & 44 & 36.7 \\
\hline
\end{tabular}

Table 1 shows that females were the majority (65.8\%) and males were $34.2 \%$ respectively. The table further shows the employment status with $63.3 \%$ of the respondents employed and only $36.7 \%$ were unemployed. The table also shows that there was equal representation of victims and perpetrators of domestic violence with $50.0 \%$ each respectively. Finally, although not included in the table participant's age range was 18-56 years with majority of the respondents in between 2230 years of age. 
Table 2. Descriptive Statistics in relation to Gender (males and females) and Role (victims and perpetrators) on Sub-Scales on Personality Traits (N=120).

\begin{tabular}{|c|c|c|c|c|c|}
\hline & Gender & Role in Domestic Violence & Mean & Standard Deviation & $\mathbf{N}$ \\
\hline \multirow{4}{*}{ Extroversion } & \multirow{2}{*}{ Male } & Victim & 2.8571 & 1.35062 & 14 \\
\hline & & Perpetrator & 3.2593 & 1.74516 & 27 \\
\hline & \multirow{2}{*}{ Female } & Victim & 4.0000 & 2.16025 & 46 \\
\hline & & Perpetrator & 3.3167 & 1.43198 & 60 \\
\hline \multirow{4}{*}{ Neuroticism } & \multirow{2}{*}{ Male } & Victim & 4.5000 & 2.71038 & 14 \\
\hline & & Perpetrator & 5.7037 & 2.70064 & 27 \\
\hline & \multirow[t]{2}{*}{ Female } & Perpetrator & 5.3939 & 2.03008 & 33 \\
\hline & & Perpetrator & 5.5333 & 2.33954 & 60 \\
\hline \multirow{5}{*}{ Psychoticism } & \multirow{2}{*}{ Male } & Victim & 3.0714 & 1.63915 & 14 \\
\hline & & Perpetrator & 2.4074 & 1.59950 & 27 \\
\hline & \multirow{3}{*}{ Female } & Victim & 2.7609 & 1.64904 & 46 \\
\hline & & Perpetrator & 3.2121 & 1.78111 & 33 \\
\hline & & Perpetrator & 2.8500 & 1.73523 & 60 \\
\hline
\end{tabular}

Table 2 shows role in Domestic violence scores personality traits scales result in relation to extroversion show that male victims had lower scores on extroversion compared to the female victims, whose mean scores were high. Moreover, victims had higher scores on extroversion than perpetrators. Gender scores on personality In addition, female victims had higher mean scores on neuroticism. However, male perpetrators had higher mean scores than females but, overall, perpetrators had higher mean scores than victims. Also, the standard deviations suggest there is imaginative variance. Psychoticism results show that male victims had higher mean scores than female victims. However, female perpetrators scored higher on psychoticism than did males. Overall, perpetrators scored higher than victims in relation to psychoticism personality traits. This implies that perpetrators are had to condition to associate anxiety with perpetrating violence.

\subsection{Inferential Results}

Table 3. A Multivariate Testa.

\begin{tabular}{|c|c|c|c|c|c|c|c|c|c|}
\hline Effect & & Value & $\mathbf{F}$ & Hypothesis df & Error df & Sig. & $\begin{array}{l}\text { Partial Eta } \\
\text { Squared }\end{array}$ & $\begin{array}{l}\text { Noncent } \\
\text { Parameter }\end{array}$ & $\begin{array}{l}\text { Observe } \\
\text { d Power }\end{array}$ \\
\hline \multirow{4}{*}{ Intercept } & Pillai's Trace & 512 & $39.526 b$ & 3.000 & 113.000 & .000 & .512 & 118.579 & 1.000 \\
\hline & Wilks' Lambda & .488 & $39.526 b$ & 3.000 & 113.000 & .000 & .512 & 118.579 & 1.000 \\
\hline & Hotelling's Trace & 1.049 & $39.526 b$ & 3.000 & 113.000 & .000 & .512 & 118.579 & 1.000 \\
\hline & Roy's Largest Root & 1.049 & $39.526 b$ & 3.000 & 113.000 & .000 & .512 & 118.579 & 1.000 \\
\hline \multirow{3}{*}{ LIE } & Pillai's Trace & .067 & $2.711 \mathrm{~b}$ & 3.000 & 113.000 & .048 & .067 & 8.133 & .645 \\
\hline & Wilks' Lambda & .933 & $2.711 \mathrm{~b}$ & 3.000 & 113.000 & .048 & .067 & 8.133 & .645 \\
\hline & Roy's Largest Root & .072 & $2.711 \mathrm{~b}$ & 3.000 & 113.000 & .048 & .067 & 8.133 & .645 \\
\hline \multirow{4}{*}{ Gender } & Pillai's Trace & .035 & $1.368 \mathrm{~b}$ & 3.000 & 113.000 & .256 & .035 & 4.103 & .356 \\
\hline & Wilks' Lambda & .965 & $1.368 \mathrm{~b}$ & 3.000 & 113.000 & .256 & .035 & 4.103 & .356 \\
\hline & Hotelling's Trace & .036 & $1.368 \mathrm{~b}$ & 3.000 & 113.000 & .256 & .035 & 4.103 & .356 \\
\hline & Roy's Largest Root & .036 & $1.368 \mathrm{~b}$ & 3.000 & 113.000 & .256 & .035 & 4.103 & .356 \\
\hline \multirow{3}{*}{ Role } & Pillai's Trace & .019 & $.736 b$ & 3.000 & 113.000 & .533 & .019 & 2.207 & .203 \\
\hline & Wilks' Lambda & .981 & $.736 b$ & 3.000 & 113.000 & .533 & .019 & 2.207 & .203 \\
\hline & Roy's Largest Root & .020 & $.736 b$ & 3.000 & 113.000 & .533 & .019 & 2.207 & .203 \\
\hline \multirow{4}{*}{$\begin{array}{l}\text { Gender * } \\
\text { Role }\end{array}$} & Pillai's Trace & .041 & $1.629 b$ & 3.000 & 113.000 & .187 & .041 & 4.888 & .418 \\
\hline & Wilks' Lambda & .959 & $1.629 \mathrm{~b}$ & 3.000 & 113.000 & .187 & .041 & 4.888 & .418 \\
\hline & Hotelling's Trace & .043 & $1.629 \mathrm{~b}$ & 3.000 & 113.000 & .187 & .041 & 4.888 & .418 \\
\hline & Roy's Largest Root & .043 & $1.629 \mathrm{~b}$ & 3.000 & 113.000 & .187 & .041 & 4.888 & .418 \\
\hline
\end{tabular}

a. Design: Intercept + LIE + Gender + Role + Gender * Role.

b. Exact statistic.

c. Computed using alpha $=.05(\mathrm{p}<.04$ Significant on Bonferroni correction $)$.

MANCOVA Results on Personality traits. The assumption of homogeneity is met since $\mathrm{p}=.125>.04$ (Bonferroni adjustment alpha) for personality traits is satisfied. Thus table 3 presents the multivariate results revealed by Pillai Traces ( $p$ $=.256$ which is bigger than .04) show that there are no gender differences regarding personality traits. Similarly, there are no significant role differences, revealed by $\mathrm{p}=.533$ which is bigger than .04. This implies that a null hypothesis is accepted that gender and role groups are not significantly different on personality traits score based on MANOCVA derived combined dependent variables together in a canonical manner. However, through examining effect .019 for role and .035 for gender, in line with Cohen's guidelines [23], it is observed that the partial Eta Squared - small effect 
size for gender (.035) and small effect size for role (.019) shows variability in impact across all dependent variables within gender and role groups. Moreover, personality traits levene's test of equality of error variance results satisfied the assumption of homogeneity (imaginative variance) at 0.04 Bonferroni adjustment alpha. This allows to present and discuss next the univariate tests of between-subject's effects which examine each dependent variable.

Table 4. Tests of Between-Subjects Effects.

\begin{tabular}{|c|c|c|c|c|c|c|c|c|c|}
\hline Source & $\begin{array}{l}\text { Dependent } \\
\text { Variable } \\
\end{array}$ & $\begin{array}{l}\text { Type } 111 \text { Sum of } \\
\text { Squares }\end{array}$ & Df & $\begin{array}{l}\text { Mean } \\
\text { Square }\end{array}$ & $\mathbf{F}$ & Sig. & $\begin{array}{l}\text { Partial Eta } \\
\text { Squared }\end{array}$ & $\begin{array}{l}\text { Noncent } \\
\text { Parameter }\end{array}$ & $\begin{array}{l}\text { Observed } \\
\text { Power } \\
\end{array}$ \\
\hline \multirow{3}{*}{$\begin{array}{l}\text { Corrected } \\
\text { Model }\end{array}$} & Extroversion & $21.128 \mathrm{i}$ & 4 & 5.282 & 1.722 & .150 & .057 & 6.887 & .513 \\
\hline & Neuroticism & $38.965 \mathrm{j}$ & 4 & 9.741 & 1.689 & .157 & .055 & 6.757 & .505 \\
\hline & Psychoticism & $14.078 \mathrm{k}$ & 4 & 3.520 & 1.257 & .291 & .042 & 5.029 & .383 \\
\hline \multirow{3}{*}{ Intercepted } & Extroversion & 119.087 & 1 & 119.087 & 119.087 & .000 & .252 & 38.818 & 1.000 \\
\hline & Neuroticism & 203.581 & 1 & 203.581 & 203.581 & .000 & .235 & 35.303 & 1.000 \\
\hline & Psychoticism & 73.809 & 1 & 73.809 & 73.809 & .000 & .187 & 26.367 & .999 \\
\hline \multirow{3}{*}{ LIE } & Extroversion & 1.739 & 1 & 1.739 & .567 & .453 & .005 & .567 & .116 \\
\hline & Neuroticism & 25.152 & 1 & 25.152 & 4.362 & .039 & .037 & 4.362 & .544 \\
\hline & Psychoticism & 3.418 & 1 & 3.418 & 1.221 & .271 & .011 & 1.221 & .195 \\
\hline \multirow{3}{*}{ Gender } & Extroversion & 9.567 & 1 & 9.567 & 3.118 & .080 & .026 & 3.118 & .417 \\
\hline & Neuroticism & 2.204 & 1 & 2.204 & .382 & .538 & .003 & .382 & .094 \\
\hline & Psychoticism & 1.453 & 1 & 1.453 & .519 & .473 & .004 & .519 & .110 \\
\hline \multirow{3}{*}{ Role } & Extroversion & .226 & 1 & .226 & .074 & .786 & .001 & .074 & .058 \\
\hline & Neuroticism & 10.887 & 1 & 10.887 & 1.888 & .172 & .016 & 1.888 & .276 \\
\hline & Psychoticism & .144 & 1 & .144 & .051 & .821 & .000 & .051 & .056 \\
\hline \multirow{3}{*}{$\begin{array}{l}\text { Gender * } \\
\text { Role }\end{array}$} & Extroversion & 6.755 & 1 & 6.755 & 2.202 & .141 & .019 & 2.202 & .313 \\
\hline & Neuroticism & 9.814 & 1 & 9.814 & 1.702 & .195 & .015 & 1.702 & .253 \\
\hline & Psychoticism & 7.695 & 1 & 7.695 & 2.749 & .100 & .023 & 2.749 & .376 \\
\hline \multirow{3}{*}{ Error } & Extroversion & 352.797 & 115 & 3.068 & & & & & \\
\hline & Neuroticism & 663.160 & 115 & 5.767 & & & & & \\
\hline & Psychoticism & 321.914 & 115 & 2.799 & & & & & \\
\hline \multirow{3}{*}{ Total } & Extroversion & 1865.000 & 120 & & & & & & \\
\hline & Neuroticism & 4169.000 & 120 & & & & & & \\
\hline & Psychoticism & 1305.000 & 120 & & & & & & \\
\hline \multirow{3}{*}{$\begin{array}{l}\text { Corrected } \\
\text { Total }\end{array}$} & Extroversion & 373.925 & 119 & & & & & & \\
\hline & Neuroticism & 702.125 & 119 & & & & & & \\
\hline & Psychoticism & 335.992 & 119 & & & & & & \\
\hline
\end{tabular}

a. $\mathrm{R}$ Squared $=.057$ (Adjusted R Squared $=.024)$.

b. $\mathrm{R}$ Squared $=.055$ (Adjusted R Squared $=.023$ ).

c. R Squared $=.042$ (Adjusted R Squared $=.009$ ).

d. Computed using alpha $=.05(\mathrm{p}<.04$ (Significant on Bonferroni correction).

Table 4 presents the MANCOVA personality results for all dependent variables-traits sub-scales in relation to gender and role and the interaction between the two, while controlling lie as a covariate. The results of interest are those in the rows relating to gender, role and their interaction between gender and role. Firstly, the univariate results in relation to gender show no significant gender differences in relation to all dependent variables ( $\mathrm{p}$ values are bigger than .04 Bonferroni adjusted alpha). Hence, it can be concluded that males and females, did not report significant differences in personality traits scores on: neuroticism, extroversion and psychoticism

Secondly, results in relation to role show significant differences between victims and perpetrators concerning neuroticism, extroversion and psychoticism personality traits scores ( $\mathrm{p}$ values are bigger than $>.04$ Bonferroni adjusted alpha). Results show no interaction between role and gender in relation to personality traits scores, domestic violence subscales.

Overall, a two by two MANCOVA was conducted to explore the impact of role and gender on personality traits sub-scales: Extroversion, Neuroticism and Psychoticism were dependent variables. The independent variables were role (victims and perpetrators) and gender (males and females). Preliminary tests were conducted to check for normality, linearity, univariate, multivariate and homogeneity and no serious violations noted. The results show no statistically significant difference and no interaction between males and females, victims and perpetrators on all dependent variables (for gender $\mathrm{F}=.1 .368, \mathrm{P}=.256$; Pillai Trace $=.035$; partial eta squared $=.035$ ); for role $\mathrm{F}=.736, \mathrm{P}=.533$; Pillai Trace $=.019$; partial eta squared $=.019$ ). When the results for dependent variables were considered separately, there was no difference of statistical significance using a Bonferroni adjusted alpha level of .016 .

\section{Discussion}

Personality traits show no statistically role and gender significant differences and no interaction on all personality traits. However, more perpetrators more than victims reported slightly higher scores on neuroticism trait and psychoticism than victims, who only had a higher mean on the extroversion scale. These findings on personality traits do not challenge existing understandings of personality in 
previous studies but rather partly agree with them. For example, the Ugandan study concurs with a large study that investigated people from 50 cultures from all continents except Antarctica, in which participants evaluated someone they knew well on traits using the 'Big five questionnaires [14]. This is particularly important as it bolsters the findings of McCrae's study in respect of some developing countries (e.g., Botswana, Ethiopia, Lebanon, Malaysia, Puerto Rico and Uganda) where the evidence was weakest and suggested that people may not have fully understood the questions or were unfamiliar with answering questions in that format [24].

Hence, despite personality traits not correlating with most other variables, generally there were more statistically significant results reported by victims' findings than perpetrators' within the Ugandan sample. Generally, female perpetrators scored highly on personality traits. For example, females rated highly on neuroticism and psychoticism personality traits and male perpetrators rated highly on extroversion trait. However, extroversion and psychoticism traits were rated highly by more female victims than male victims. These results contradict with [25] findings on selfrating carried out using the Big Five Inventory in 56 nations. The direct comparisons of the self-report means [26], from across these countries suggest that, for example, that 'the world champions of neuroticism are Spaniards, the most extroverted people in the world are from Denmark, the nationality that is was found to be open to new experiences were Australians where as the most agreeable people in the world are Malaysians, and the world's least conscientious nation of people is Japan' [14]. Contrary, [14]. noted that the replication of Big five in 50 developing countries, including Uganda, Botswana, Ethiopia, was questionable because of potential cultural misunderstandings of the phrasing of some questions. Hence, in this study, Eysenck's personality questionnaire (which has been validated in African contexts) was used. The findings concur with those from earlier research [27] which showed that people who score high score on neuroticism are for example (moody, emotional, low selfesteem-low scorers are stable and unreactive), psychoticism high scorers are (unempathetic, tough minded, impulsive, anti-social, impersonal, cold and egocentric) and extroversion higher scorers (dominant, active, assertive, care free). Hence, this study contributes to the knowledge on the personality characteristics of victims and perpetrators of domestic violence by examining these factors within a Ugandan sample.

Firstly, findings revealed no gender differences and role differences on sub-scales of personality traits. In addition, although there was no interaction between gender and role in domestic violence, through inspecting mean scores, perpetrators more than victims reported slightly higher scores on Neuroticism (implying that they are anxious, depressed and react slowly to aversive stimuli but also have high levels of stability); and psychoticism (higher scorers are considered aggressive, antisocial, cold and egocentric). Contrary, study results show that irrespective of gender, victims more than perpetrators had high scores on extraversion. Although, majority of the victims were females, it is not surprising that in the African-Ugandan context, females reported higher scores on extroversion. Higher scores on extroversion according to [5], implies that females are more social, lively and sensation seeking. Thus, this study informs counselling psychologists, policy makers and practitioners of insignificant gender and role differences regarding personality traits scores on extroversion, neurotic or psychotic. Similarly, [28], study found that Big Five Factor Model does not differ by sex. However, mean level differences were detected.

Secondly, regarding why such misunderstandings may have happened/occurred, I reflect back on the argument by [14] that culture is quite clearly implicated in people's selfconcepts and personalities. Similarly, in studies by [29], the results have largely contrasted cultures on measures of the five factor model of personality. This large body of research raises questions regarding personality across cultures- are personality factors similar across people from different cultures? Or, alternatively, does the factor model or other personality measures, such as the Eysenck personality questionnaire, reflect ideas about personhood that are limited to the West, where vast majority of this research has been conducted (Fiske et al. 2010). These questions are not new and are the basis of the replication of 'Big five' research which has been carried out with people in dozens of cultures around world, including within developing countries such as Uganda, Botswana, Ethiopia, Lebanon, and Malaysia [29]. For instance, Eysenck's personality traits recommends a Ugandan indigenous personality measure, but this must be tested for robustness. This is because previous studies exploring personality traits of victims and perpetrators of both genders [30]. This raises questions as to whether or not domestic violence and coercive behaviours are related to an individual's personality trait scores [30].

Thirdly, the implications of this study results for Understanding the risk of violence women face from other women in polygamous marriages is paramount. For instance, the implications of the study's findings on the existence of female perpetrators of domestic violence can be discussed in relation to a previous study on criminal homicide in Uganda [31]. Mushanga argued that women who perpetrate violence to other women may have been victims at the hands of males, and who then take their revenge on those they consider are the cause of their misery-primarily co-wives in polygamous marriages. Mushanga provides examples of a wife killing a co-wife in slain polygamous families and argues that often, conflict will become serious if the husband does not exercise impartially and equity in distribution of income according to socially accepted principles, for example, the most senior wife gets more, as do wives with more children is distributed accordingly [31]. Favouritism and preferential treatment, even if merely suspected, can lead to jealousy, fights, quarrels among co-wives [31]. Mushanga also notes that in polygamous families disputes and physical violence erupts between co-wives, between sons and their step-mothers and, between wives as a group against the husband [31]. 
Consequently, though a man in such cases can be a victim of female violence, this cannot be separated from the wider gendered context in which the nature of power in patriarchal societies and the attitudes it promulgates give men the right to control women and bound women to the rules put in place by men. Polygamous households are prevalent in Uganda. According to [32], 25 percent of women in Uganda are in polygynous unions-marriages with two or more co-wives. In addition, 17 percent of men aged 15-54 in Uganda reported having two or more wives - a level that had remained constant for the previous five years [32]. Despite this, there is little recognition of this issue in domestic violence policy. This current study opens up new angles to exploring gender relations in domestic violence cases in polygamous marriages in Uganda and fills a void in the research available. In Uganda, like in Turkey, a study by [33] revealed that Turkish women also scored higher in Neuroticism than other groups. Previous research [33] recommends that professionals working with female victims should help them ameliorate neuroticism to reduce risks of depressive symptoms.

\subsection{Conclusion}

In summary, a two by two MANCOVA was conducted to explore the impact of role and gender on personality traits subscales: Extroversion, Neuroticism and Psychoticism were dependent variables. The independent variables were role in GBV (victims and perpetrators) and gender (males and females). Preliminary tests were conducted to check for normality, linearity, univariate, multivariate and homogeneity and no serious violations noted. Basing on results from victims and perpetrators of Gender Based Violence, results show no statistically significant differences and no interaction between males and females, victims and perpetrators regarding Extroversion, Neuroticism and Psychoticism personality traits. However, perpetrators more than victims reported slightly higher mean scores on Neuroticism and psychoticism. In contrast, victim's results revealed that irrespective of gender, victims more than perpetrators had high scores on extraversion. This study findings on personality differences although not significant informs counsellors, psychologists and other helping professionals the need for gender inclusivity approach in handling cases of domestic violence and also being aware of individual differences.

\subsection{Limitations of the Study}

This study lacked a non-victim and non perpetrator comparison group. As a result, the findings, while providing original knowledge, are limited in being able to assess selfreported victimisation, victimisation reported by non-victim and non-perpetrator comparison groups. This is limiting in a sense that non-victim and non perpetrator comparison group results are missed.

\section{Funding}

This study didn’t receive any funding.

\section{References}

[1] Maltby, J, Day, L, Macaskill, A, Hall, P. (2007). Personality, Individual Differences and Intelligence. Personality and individual differences 43 (2), 341-351, 2007. 246, 2007.

[2] McCrae, R. R. (2000). Trait Psychology and the revival of Personality and Culture studies. American Behavioral Scientists 44, 10-31.

[3] Feist, J., \& Feist, G. J. (2009). Theories of Personality, Seventh Edition. International.

[4] Ewen, R. B. (1998). Personality: A topical approach: Theories, research, major controversies, and emerging findings. Mahwah, NJ, US: Lawrence Erlbaum Associates Publishers.

[5] Mathews, M., Deary I. J., \& Whiteman, M. C. (2009). Personality traits, 3rd edn Cambridge, UK: Cambridge University Press.

[6] Costa, P. T., \& McCrae, R. R. (2003). Personality in Adulthood. A five-factor theory Perspective (2nd Ed.) New York: Guilford Press.

[7] Eysenck, H. J (Ed). (1981). A model for personality. New York: Springer.

[8] Zhang, L. (2006). Thinking styles and the big five personality traits revisited. Personality and Individual differences.

[9] Howitt, D. (2009). Introduction to forensic and Criminal Psychology. Pearson Education, ISBN 978-1-4058-7446-5.

[10] Berkowitz, L. (1993). Aggression: Its causes, consequences, and control. New York: McGraw Hill.

[11] Tibatemwa, L, E. (1999). More Sinned Against than Sinning. Women's Violent Crime in Uganda. Published PhD thesis. Fountain Publishers Kampala Uganda ISBN 9970-02-166-4.

[12] Eysenck, H. J. (1996). Personality and crime: Where do we stand? Psychology, Crime and Law, 2, 143-152.

[13] Francis, L. J., Lewis, C. A., Ziebertz, H. G. (2006) 'The Shortform revised Eysenck Personality Questionnaire (EPQ-S): A German edition'. Social Behaviour and Personality, 34 (2), 197-204.

[14] Fiske, S. T., Gilbert, D. T., \& Lindsey, G. (Eds) (2010). Handbook of Social Psychology (5th ed) New York: Wiley.

[15] Lynn, R., \& Martin, T. (1997). Gender Differences in Extroversion, Neuroticism and Psychoticism in 37 Nations, Journal of Social Psychology, 137: 3, 369-373.

[16] Eysenck, H. J (Ed). (1964). Manual of the Eysenck Personality Inventory. London University of London Press.

[17] Black, T. R. (1999). Doing quantitative Research in the Social Sciences: An integrated approach to Research design, Measurement, and Statistics. London Sage.

[18] Eysenck, H. J., \& Eysenck, S. B. G. (1976). Psychoticism as a dimension of personality. New York: Crane, Russak \& Co.

[19] Jackson, C. J \& Francis, L. J. (1999). Interpreting the Correlation Neuroticism and Lie Scale Scores. Personality and Individual differences, 26, 59-63. 
[20] UNCST, (2014). National Guidelines for Research involving Humans as Research Participants. UNCST Bulletin, 2014.

[21] Field, A. (2000). Discovering Statistics using SPSS for Windows. SAGE Publication Ltd; ISBN-13-978-0761957553.

[22] Field, A. (2013). Discovering Statistics using IBM SPSS Statistics. SAGE Publication Ltd; Fourth Edition. ISBN-101446249182 .

[23] Pallant, J. (2013). SPSS Survival Manual: A step by step guide to data analysis using IBM SPSS, 5th Edition, Spiralbound-1 May 2013.

[24] Gilbert, D, T, Fiske, S. T, \& Lindsey, G. (2010). Handbook of social psychology (5th ed., pp. 668-697).

[25] Schmitt, D. P., Allik, J., McCrae, R. R., Benet-Martínez, V., Alcalay, L., \& Ault, L. (2007). The geographic distribution of Big Five personality traits: Patterns and profiles of human self description across 56 nations. Journal of Cross-Cultural Psychology, 38, 173-212. doi: 10.1177/ 0022022106297299.

[26] McCrae, R. R. (2002). NEO-PI-R data from 36 cultures: Further intercultural comparisons. In R. R. McCrae \& J. Allik (Eds.), The Five-Factor Model of personality across cultures (pp. 105-126). New York: Kluwer Academic/Plenum Publishers.

[27] Eysenck, S. B. G., Eysenck, H. J., \& Barrett, P. (1985). A revised version of the Psychoticism scale. Personality and Individual Differences, 6, 21-29.
[28] Susan C. S., Amber M. J., \&, Colin E. V (2018). Sex differences in the Big Five model personality traits: A behavior genetics exploration. Journal of Research in Personality 74 (2018) 158-165.

[29] McCrae, R. R. \& Terracciano, A., (2005). The 78 Members of the Personality Profiles of Cultures Project. (2005a). Universal features of personality traits from the observer's perspective: Data from 50 cultures. Journal of Personality and Social Psychology, 88, 547-561.

[30] Wyrod, R. (2007). Bwaise Town: Masculinity in urban Uganda. Age of AIDS. Doctoral Thesis. University of Chicago, Chicago.

[31] Mushanga, T. M. (2013). Criminal Homicide in Uganda. A Sociological study of violent Death, Ankole, Kigezi and Toro Districts of Western Uganda. Law Africa Publishing (U) Ltd.

[32] Uganda Bureau of Statistics and Macro International, Inc (UBOS, 2011). Uganda Demographic and Health Survey, Calverton, MD: UBOS 2011.

[33] Silvaggi M, Maria Michetti P, Rossi R, Fabrizi A, Leonardo C, et al. (2017) Extraversion and Neuroticism in Sexually Dysfunctional Men Suffering from Erectile Dysfunction and Premature Ejaculation: A Cross-Sectional Study. Andrology (Los Angel) 6: 1000181. doi: 10.4172/2167-0250.1000181.

[34] Timo, J. (2018). Sex-Free and Sex-Related Components of the Eysenck Personality Questionnaire (EPQ) Neuroticism Scale among Finnish and Turkish Students. Journal of Social Sciences, 2018, 7, 38. 